\title{
Práticas fisioterapêuticas para o tratamento das disfunções temporomandibulares: avaliação do conhecimento de cirurgiões-dentistas
}

\section{Physiotherapeutic practices for the treatment of temporomandibular disorders: evaluation of the knowledge of dentists}

\author{
Rafaela Souto Colares, Lidia Audrey Rocha Valadas Marques, \\ Mariana Lima Fernandes, Camila Costa Dias, \\ Karoline Sampaio Nunes Barroso, Thiago Guedes Teles
}

Como citar este artigo: COLARES, RAFAELA S.; MARQUES, LIDIA A. R. V.; FERNANDES, MARIANA L.; DIAS, CAMILA C.; BARROSO, KAROLINE S. N.; TELES, THIACO C. Práticas fisioterapêuticas para o tratamento das disfunções temporomandibulares: avaliação do conhecimento de cirurgiõesdentistas. Revista Saúde (Sta. Maria). 2020; 46 (2).

\footnotetext{
Autor correspondente: Nome: Rafaela Souto Colares

E-mail: rafaela.s.colares@hotmail.com Telefone: (85) 988419195

Formação Profissional: Formadaem Fisioterapia pela Faculdades Nordeste (FANOR) que fica na cidade de Fortaleza, estado CE, Brasil

Filiação Institucional: Hospital Distrital Dr. Fernandes Távora Endereco para correspondencia: Avenida Francisco Sá

Bairro: Alvaro Weyne

Cidade: Fortaleza

Estado: Ceará

CEP: 60335-195
}

Data de Submissão:

25/03/2020

Data de aceite:

$21 / 09 / 2020$

Conflito de Interesse: Não há conflito de interesse

\section{(cc) $\mathrm{BY}-\mathrm{NC}-\mathrm{ND}$}

\section{RESUMO}

Introdução: A disfunção temporomandibular (DTM) é uma das condições orofaciais mais prevalentes e por ser multifatorial, necessita de tratamento multidisciplinar. É frequente o número de pessoas que apresentam sinais ou sintomas de DTM, porém, poucas são conhecedoras desta disfunção e buscam por tratamento. A falta de comunicação multidisciplinar ou a ausência de conhecimento por parte dos cirurgiões-dentistas em relação aos fisioterapeutas, muitas vezes impedem o tratamento multidisciplinar. 0 objetivo da presente pesquisa foi investigar o conhecimento dos cirurgiões-dentistas sobre a atuação da Fisioterapia no tratamento das DTM. Metodologia: Trata-se de um estudo quantitativo, do tipo analítico transversal. Foram investigados 29 cirurgiões-dentistas que trabalhavam no setor privado e/ou público, com idades entre 25 e 60 anos, de ambos os gêneros. Resultados: Os resultados da pesquisa mostraram que não existe um encaminhamento eficiente dos pacientes com DTM por parte dos cirurgiões-dentistas aos fisioterapeutas, para que assim ocorra um tratamento mais eficaz. Foi constatado que essa situação não ocorria por falta de conhecimento dos cirurgiões-dentistas $(83,0 \%)$, sobre a atuação da Fisioterapia. Apenas 38\% já tinham feito esse tipo de encaminhamento. Entre os mesmos $97 \%$ acreditam na importância do tratamento multidisciplinar. No entanto, os entrevistados ressaltaram a sua opinião sobre a importância da comunicação entre as áreas envolvidas, porém não manifestaram a razão da falta de encaminhamentos e da integração entre os profissionais. Entre os participantes $48 \%$ afirmaram ter sido na graduação o primeiro contato com esse tipo de informação interdisciplinar. Conclusão: Poucos cirurgiões-dentistas encaminham os pacientes com DTM para os fisioterapeutas, portanto faz-se necessário divulgar mais resultados das evoluções de casos clínicos para a área da Odontologia, além de realizar pesquisas mais focadas em concretizar pensamentos e atitudes interdisciplinares entre os profissionais da saúde de forma geral dentro do tratamento da DTM.

PALAVRAS-CHAVE: Fisioterapia; Práticas interdisciplinares; Odontologia.

\section{ABSTRACT}

Introduction: Temporomandibular disorder (TMD) is one of the most prevalent orofacial conditions and because it is multifactorial, it requires multidisciplinary treatment. The number of people with TMD signs or symptoms is frequent, however, few are aware of this dysfunction and seek treatment. The lack of multidisciplinary communication or the lack of knowledge on the part of dentists in relation to physical therapists, often prevent multidisciplinary treatment. The aim of this research was to investigate the knowledge of dentists on the role of Physiotherapy in the treatment of TMD. Methodology: This is a quantitative, analytical cross-sectional study. 29 dental surgeons who worked in the private and / or public sector, aged between 25 and 60 years, of both genders, were investigated. Results: The research results showed that there is no efficient referral of patients with TMD by dentists to physiotherapists, so that a more effective treatment occurs. It was found that this situation did not occur due to the lack of knowledge of dentists (83.0\%) about the role of Physiotherapy. Only 38\% had already made this type of referral. Among the same $97 \%$ believe in the importance of multidisciplinary treatment. However, the interviewees emphasized their opinion on the importance of communication between the areas involved, however, they did not express the reason for the lack of referrals and integration among professionals. Among the participants, $48 \%$ stated that their first contact with this type of interdisciplinary information was during graduation. Conclusion: Few dental surgeons refer patients with TMD to physiotherapists, therefore it is necessary to disclose more results from the evolution of clinical cases in the area of Dentistry, in addition to conducting more focused research to concretize interdisciplinary thoughts and attitudes among professionals in the field. general health within the treatment of TMD. 


\section{INTRODUÇÃO}

A articulação temporomandibular (ATM) é formada entre o osso temporal e a mandíbula, além de estruturas associadas, como o disco, cápsula articular, ligamentos, músculos e fluido sinovial. Essa articulação nos permite falar, deglutir, mastigar e alimentar-se devido aos movimentos de abertura e fechamento da boca. Além disso, é considerada umas das articulações mais complexas do corpo humano ${ }^{1,2}$.

Por ser uma articulação bastante complexa, a ATM é frequentemente acometida por disfunções, sendo estas multifatoriais, causadas por traumatismos diretos, acidentes automobilísticos, quedas, maus hábitos posturais e hábitos parafuncionais os quais constituem alguns exemplos de fatores que ocasionam a Disfunção Temporomandibular (DTM). Vários fatores estão relacionados ao desequilíbrio funcional do aparelho estomatognático ou da biomecânica da ATM, e também por predispor às DTM, cujos principais sinais e sintomas são a dor e os ruídos articulares ${ }^{3,4}$.

As DTM podem ser explicados como um conjunto de condições dolorosas, e/ou disfuncionais, que englobam os músculos da mastigação e/ou as articulações temporomandibulares ${ }^{5}$.

Segundo Donnarumma et al. (2008) $)^{6}$ a DTM deve ter uma abordagem multiprofissional, a qual permitirá uma maior e um tratamento mais efetivo. De acordo com as necessidades do paciente, várias especialidades podem atuar nisso, como: Odontologia, Fisioterapia, Fonoaudiologia, Psicologia e Medicina. Logo, a multidisciplinaridade entre estes profissionais seria o mais indicado para proporcionar um melhor acompanhamento aos pacientes portadores de DTM.

De acordo com Fernandes et al. (2014) ${ }^{7}$, a atuação do tratamento fisioterapêutico procura restabelecer a ATM que está comprometida, tendo como objetivo inverter essa disfunção. E assim, com um atendimento multidisciplinar, controlam-se os sinais e sintomas que se manifestam nessa disfunção.

Para restabelecimento da articulação, existem diversos tipos de tratamento que a Fisioterapia dispõe em suas modalidades, como o uso de técnicas de relaxamento muscular, além do fortalecimento e da utilização da eletrotermofototerapia para uma melhor adaptação desta ${ }^{8}$. Sendo assim, o presente estudo buscou investigar o conhecimento dos cirurgiões-dentistas sobre a atuação da Fisioterapia no tratamento das DTM, além de identificar a frequência de encaminhamentos de pacientes com DTM, por parte dos cirurgiões-dentistas para fisioterapeutas.

\section{MÉTODOS}

\section{Tipo e local de estudo}

Trata-se de um estudo de abordagem predominantemente quantitativa, do tipo analítico e transversal. A pesquisa foi realizada em consultórios odontológicos e instituições públicas da cidade de Fortaleza-CE, seguindo a resolução 466/12 sobre pesquisa com seres humanos. 


\section{Amostra da pesquisa}

Cirurgiões-dentistas do estado do Ceará que trabalham no setor privado e/ou público, com idades entre 25 e 60 anos, de ambos os gêneros, aceitaram a participação na pesquisa.

\section{Critérios de inclusão e exclusão}

Como critérios de inclusão, participaram da pesquisa os profissionais odontólogos regularmente registrados no Conselho Regional de Odontologia (CROCE) e que trabalham pelo menos um período do dia em consultório odontológico (setor privado) e/ou posto de saúde (setor público).

Foram excluídos da pesquisa os profissionais com menos de um ano de serviço no setor público ou privados, e profissionais que não atenderam a nenhum paciente com DTM.

\section{Coleta e análise de dados}

Após assinatura do Termo de Consentimento Livre e Esclarecido (TCLE) foi aplicado aos cirurgiões-dentistas selecionados, um questionário semiestruturado, com questões objetivas, com intuito de investigar o conhecimento desses profissionais sobre a atuação da Fisioterapia no tratamento das DTM. Os resultados foram tabulados no Microsoft Excel, descritos e apresentados em forma de tabelas.

\section{RESULTADOS}

Entre os entrevistados 62,0\% (18) eram do gênero feminino, 35,0\%(10) do gênero masculino e 3,0\%(1) não responderam. A média de idade foi 37,5 anos. Quanto ao tempo de profissão dos cirurgiões-dentistas no atendimento ao setor público e privado, percebeu-se uma predominância de profissionais com até 10 anos de experiência profissional $(55,0 \%)$.

Quanto o tipo de serviço que os participantes da pesquisa atuam, se no setor particular, público ou em ambos, predominou o serviço público $(38 \%, 0)$. Grande parte dos profissionais $(83,0)$ afirmaram possuir conhecimento quanto a atuação da Fisioterapia no tratamento multidisciplinar das DTM (Tabela 01).

Notou-se que $48 \%$ dos cirurgiões-dentistas afirmaram obter informações, ainda durante o curso de graduação, sobre a atuação da Fisioterapia no tratamento das DTM. Já os outros $52 \%$ afirmaram que não obtiveram qualquer tipo de informação a respeito (Tabela 01).

Ainda na tabela 01 observa-se que $97 \%$ dos consideram importante a comunicação entre os profissionais odontólogos e fisioterapeutas no tratamento das DTM, e 3\% não responderam à questão. 
Tabela 01: Conhecimento sobre o papel da Fisioterapia na Disfunção Temporomandibular

\begin{tabular}{lll}
\hline & N & $\%$ \\
\hline Tipo de atendimento & 11 & 38,0 \\
Público & 8 & 28,0 \\
Particular & 9 & 31,0 \\
Público e Particular & 1 & 3,0 \\
Não responderam & & \\
Conhecimento sobre atuação da & & \\
Fisioterapia no tratamento de DTM & 24 & 83,0 \\
Sim & 5 & 17,0 \\
Não & & \\
\hline Informação sobre atuação da Fisioterapia & 48,0 \\
no tratamento de DTM ainda na graduação & 14 & 52,0 \\
Sim & 15 & \\
Não & & 97,0 \\
Acreditam na importância da interdisciplinaridade & & 0,0 \\
entre as duas áreas no tratamento da DTM & 28 & 3,0 \\
Sim & 0 & \\
Não & 1 & \\
Não responderam &
\end{tabular}

Na tabela 02 pode-se observar que apenas $38,0 \%$ dos participantes já haviam realizado encaminhamentos de pacientes com DTM para profissionais fisioterapeutas. Entre os cirurgiões-dentistas, $90 \%$ afirmaram que associariam um tratamento conjunto com o fisioterapeuta, para uma melhor qualidade de vida do paciente com DTM, e apenas $10 \%$ dos entrevistados não associariam um tratamento.

Tabela 02: Experiência dos profissionais odontólogos acerca do tratamento interdisciplinar de DTM

\begin{tabular}{lll}
\hline & $\mathbf{n}$ & $\%$ \\
\hline Encaminhamento para tratamento & & \\
fisioterapêutico & 11 & 38,0 \\
Sim & 18 & 62,0 \\
Não & & \\
\hline Acreditam que a associação de tratamentos & \\
melhoram a qualidade de vida do paciente & 26 & 90,0 \\
Sim & 6 & 10,0 \\
Não & & \\
\hline
\end{tabular}




\section{DISCUSSÃO}

Os serviços da Odontologia são bastante procurados devido às dores periarticulares e suas regiões proximais, porém a origem desse desconforto nem sempre é dentária, mas sim uma desordem na ATM $^{9}$. A DTM vem chamando atenção na atuação clínica em especial dos ortodontistas durante o tratamento dos pacientes, havendo assim um cuidado em relação ao diagnóstico para uma melhor conduta, porém não se pode afirmar que o tratamento ortodôntico previne ou cura a disfunção temporomandibular ${ }^{10}$. Além disso, o principal recurso da Odontologia para as correções da sintomatologia das DTM são os dispositivos interoclusais. Quando o tratamento com esses dispositivos não geram resultados em curto prazo, o funcionamento do sistema estomatognático pode ser comprometido ${ }^{11}$. A Fisioterapia dispõe de vários recursos para o tratamento no alívio das sintomatologias e na estabilização da função da ATM, sendo também utilizados alguns recursos para restabelecer a postura, bem como a massoterapia, a cinesioterapia, a termoterapia e a eletroterapia ${ }^{12}$.

Observa-se nos resultados que grande parte dos cirurgiões-dentistas (83,0\%) tiveram, ao longo da sua trajetória, explanações sobre a atuação integrada da Fisioterapia no tratamento das DTM. Então, a falta de encaminhamento não ocorre pela falta de conhecimento ${ }^{13}$.

Estudos demonstram que a atuação da Fisioterapia é fundamental na intervenção da DTM, não apenas nas reabilitações da ATM, mas também como uma visão global, além de restabelecer as funções musculoesqueléticas ${ }^{9}$. Com o uso dos recursos fisioterápicos, é possível evitar cirurgias, fazendo com que ocorra o reposicionamento da mandíbula ao crânio, o que levaria à melhora das suas funções (mastigação, fala e bocejo), por meio da diminuição da dor muscular, além da melhora da amplitude de movimentos, o que reduziria a carga de tensão na ATM, com o fortalecimento dos músculos, fazendo não só uma reeducação postural, como também uma reposição da mandíbula12.

Segundo Pancin et al. $(2010)^{13}$, os cirurgiões-dentistas clínicos-gerais têm conhecimento sobre terapia, porém não possuem experiência no tratamento e ressalta que a evolução é apenas para o paciente com sintomatologia, portanto preferem encaminhá-los para o especialista odontólogo em ATM.

Assim, é possível perceber que, devido aos avanços técnicos e científicos observados, há a necessidade de uma interação entre as áreas envolvidas, para um melhor diagnóstico da DTM. Sendo assim, ambas se correlacionam, e é através da integração das áreas que o indivíduo recebe um atendimento mais completo e um tratamento mais globalizado. No presente estudo $97 \%$ dos participantes acreditam na importância da interdisciplinaridade com 0 profissional fisioterapêutico, entretanto somente $38 \%$ já encaminharam pacientes para esse tipo de tratamento ${ }^{14}$.

Com isso, é possível considerar que nos dias atuais há um aumento da integração das diversas áreas da saúde, o que garante ao paciente uma cura efetiva, bem como um diagnosticado com DTM, devido à intervenção conjunta entre os profissionais da Fisioterapia e da Odontologia. Portanto, torna-se cada vez mais comum a comunicação entre as 
áreas, por meio da atuação entres os profissionais, e a verificação de que as trocas de informações ocorrem desde as clínicas-escolas, traçando assim, tratamentos para as DTM ${ }^{15}$.

O sucesso da eficácia pode ocorrer devido às abordagens educacionais que venham a decorrer na oferta de explicações aos pacientes sobre o que é DTM, durante o tratamento, possibilitando a convivência dos mesmos até na avaliação da dor ou não, e de outros sintomas que possam agravar o caso ${ }^{16}$.

Portanto, é necessário que se realize um diagnóstico preciso e rápido para que se tenha um tratamento eficaz. No caso em que não for possível o diagnóstico durante a fase aguda, ocorrendo somente quando o caso se tornar crônico, haverá um maior desconforto na vida do indivíduo, o que colocará qualidade de vida do paciente em comprometimento. Entre os participantes do presente estudo, $90 \%$ acreditam que o tratamento interdisciplinar melhora a qualidade de vida do paciente ${ }^{16}$.

Com isso, pode-se sugerir que a Fisioterapia é um conjunto que não só favorece a reabilitação e a reintegração do paciente ao meio social, mas também proporciona qualidade de vida aos indivíduos ${ }^{17}$.

Estudos demonstram que é possível observar a importância do acompanhamento do cirurgião-dentista no apoio ao tratamento conjunto com o fisioterapeuta e ainda ter a possibilidade de trocas de informação entre os profissionais no objetivo de oferecer o melhor tratamento ao paciente ${ }^{18}$.

\section{CONSIDERAÇÕES FINAIS}

A maioria dos cirurgiões- dentistas acreditam na melhora da qualidade de vida no tratamento da DTM com a Fisioterapia, entretanto o encaminhamento para esse grupo de profissionais é feito com pouca frequência, portanto fazse necessário divulgar mais resultados das evoluções de casos clínicos para a área da Odontologia, além de realizar pesquisas mais focadas em concretizar pensamentos e atitudes interdisciplinares entre os profissionais da saúde de forma geral dentro do tratamento da DTM.

\section{REFERÊNCIAS}

1. Okenson JP. Tratamento das desordens temporomandibulares e oclusão. 6ed. Rio de Janeiro: Elsevier, 2008.

2. Kapandji Al. Fisiologia articular. Esquemas comentados de mecânica humana. 6 edição. Rio de Janeiro: Maloine, 2008. 
3. Norkin CC, Levangie PK. Articulações, estrutura e função: uma abordagem prática e abrangente. 2 ed. Rio de Janeiro: Revinter, 2001.

4. Martins RJ, Garcia A, Garbin CAS, Sundefeld MLM. Associação entre classe econômica e estresse na ocorrência da disfunção temporomandibular. Rev Bras Epidemiol [online], 2007; 10(2):215-22.

5. Branco RS, Branco CS, Tesch RS, Rapoport A. Freqüência de relatos de parafunções nos subgrupos diagnósticos de DTM de acordo com os critérios diagnósticos para pesquisa em disfunções temporomandibulares (RDC/ TMD). Rev. Dent. Press Ortodon. Ortop.Facial [online], 2008; 13(2):61-9.

6. Donnarumma MDC, Muzilli CA, Ferreira C, Nemr K. Disfunções temporomandibulares: sinais, sintomas e abordagem multidisciplinar. Revista CEFAC, 2010;12(5), 788-794.

7. Fernandes ML, Marques LAR, Rodrigues Neto EM, Lotif MAL, Melo FHC, Macena RHM. Profile of temporomandibular disorder patients submitted to dental and physiotherapeutic treatment at a private clinic. RSBO. Revista Sul-Brasileira de Odontologia, 2014;11:260-64.

8. Assis TO, Soares MS, Victor MM. O uso do laser na reabilitação das desordens temporomandibulares. Fisioter Mov, 2012; 25(2):453- 59.

9. Kinote APB, Monteiro LT, Vieira AAC, Ferreira NMN, Abdon APV. Perfil funcional de pacientes com disfunção temporomandibular em tratamento fisioterápico. Rev Brasileira em Promoção da Saúde, 2011;24(4):306-12.

10. Durso BC, Azevedo L R, Ferreira JTL. Inter-relação ortodontia x disfunção da articulação temporomandibular. Ibras ortodon ortop facial, 2002;7(38):155-60.

11. Vargas FF, Vargas FF, Fonseca PABD. Desordens temporomandibulares: uma abordagem fisioterapêutica e odontológica, Stomatos, 2009;15(28):,p.27-37.

12. Garcia JD, Oliveira AC. A fisioterapia nos sinais e sintomas da disfunção da articulação temporomandibular (ATM). Rev. Hórus, 2011;5(1): 1-10. 
13. Pancin AC, Monte DF, Silva PC, Pires RR, Hildebrand AS. Interdisciplinaridade entre odontologos e Fisioterapeutas no tratamento de paciente com disfunção temporomandibular na região Leme - SP. Anuário da produção Acadêmica Docente, 2010;4(7):113-24.

14. Piozzi R,Lopes FC. Desordens temporomandibulares: Aspectos clínicos e guia para odontologia e Fisioterapia. Jornal Brasileiro de Oclusão, ATM e dor orofacial, 2002; 2(5):43-7.

15. Torres F, Campos LG, Fillipini HF, Weigert KLV, Dalla GF. Efeitos dos tratamentos fisioterapêutico e odontológico em pacientes com disfunção temporomandibular. Fisioterapia em Movimento, 25(1), 117-125, 2012.

16. Campi LB, Camparis CM, Jordani PC, Gonçalves DAG. Influência de abordagens biopsicossociais e autocuidados no controle das disfunções temporomandibulares crônicas. Rev. Dor, 2013;14(3): 219-22.

17. Saar SRC, Trevizan MA. Papeis profissionais de uma equipe de saúde: visão de seus integrantes. Rev Latina-Am Enfermagem, 2007;15(1):106-12.

18. Grazia RC, Zamai CA.Alterações posturais relacionadas com disfunção temporomandibular da articulação temporomandibular e seu tratamento. Movimento \&percepção, 2006;6(8):150-62. 